Service social

\title{
L'usage des drogues et la toxicomanie, vol. I et II, Pierre Brisson, Montréal, Gaëtan Morin éditeur, 1988, 1994.
}

\section{René Auclair}

Volume 44, numéro 1, 1995

Valeurs, pratiques, action sociale

URI : https://id.erudit.org/iderudit/706687ar

DOI : https://doi.org/10.7202/706687ar

Aller au sommaire du numéro

Éditeur(s)

École de service social de l'Université Laval

ISSN

1708-1734 (numérique)

Découvrir la revue

Citer ce compte rendu

Auclair, R. (1995). Compte rendu de [L'usage des drogues et la toxicomanie, vol. I et II, Pierre Brisson, Montréal, Gaëtan Morin éditeur, 1988, 1994.] Service social, 44(1), 159-160. https://doi.org/10.7202/706687ar d'utilisation que vous pouvez consulter en ligne.

https://apropos.erudit.org/fr/usagers/politique-dutilisation/ 
le robot blanc, sans visage et sans mains, serait-il aux yeux de Groulx et Huot le travailleur social ou la travailleuse sociale de demain?

May HAZAZ

Étudiante au doctorat

École de service social

Université Laval

\section{L'USAGE DES DROGUES ET LA TOXICOMANIE, VOL. I ET II}

\section{Pierre Brisson}

Montréal, Gaëtan Morin éditeur, 1988, 1994.

Dans le premier volume l'auteur et ses collaborateurs s'intéressent en première partie au contexte général, aux approches de base et à la question des contrôles législatifs de l'usage des drogues.

L'objet de la deuxième partie porte sur les théories et les modèles explicatifs, les populations à risque et les problèmes de santé publique.

Les modèles et les réalités de la prévention, l'intervention (philosophies et pratiques), l'évaluation et la formation sont amplement décrits dans la troisième partie.

Comme il est mentionné dans la publicité, en réunissant pour une première fois en langue française un ensemble de textes qui permet à un public large de constater l'éventail des dimensions couvertes par la question des drogues, le présent ouvrage favorise une vision globale et nuancée du sujet. De plus, la perspective adoptée est résolument interdisciplinaire comme en fait foi la sélection de trente textes, représentatifs de la variété des approches comme de la diversité des points de vue sur la question.

Dans le deuxième volume l'auteur et ses collaborateurs traitent $d^{\prime}$ abord des substances et des contextes d'usage des drogues, alors que la deuxième section de l'ouvrage est orientée sur les lois, les politiques et la société.

Les populations, les milieux et la consommation sont abordés dans la troisième section; les perspectives et les pratiques de prévention le sont dans la section suivante.

Finalement, l'auteur et ses collaborateurs analysent la question des dépendances et de leurs traitements.

Ce second volume tente $d^{\prime}$ apporter des éléments de réponse aux questions mentionnées ci-dessus. 
Ce qui caractérise cet ouvrage, c'est le fait de présenter aux lecteurs une vision large et diversifiée du phénomène, englobant les dimensions scientifique et pratique; les aspects biologiques, psychologiques et sociaux; les perspectives historique, philosophique et culturelle; les intérêts politiques et économiques; les enjeux idéologiques et éthiques.

Ces volumes s'adressent non seulement aux chercheurs, mais aussi à tous les praticiens sociaux débutants et d'expérience.

René AUCLAIR

Professeur

École de service social, Université Laval

\section{LE BRUISSEMENT DU TEMPS, LE DYNAMISME DU VIEILLISSEMENT}

Marcelle Brisson

Montréal, Triptyque, 1992, 150 pages.

Cet ouvrage est un essai sur le vieillissement, une réflexion de l'auteure au mitan de sa vie, en partant du concept de l'âge. Il s'adresse à un public lettré et sera particulièrement apprécié des chercheurs en gérontologie sociale désireux de trouver une analyse théorique et développementale du vieillissement. Selon l'auteure, le vieillissement en appelle au dynamisme bio-psycho-social de l'être humain et à ses capacités de s'adapter aux différentes étapes du cycle de vie. La vieillesse catégorise les personnes et inscrit l'irréversibilité du passage du temps humain.

Cet ouvrage se partage en deux sections bien distinctes. La première partie, "Dynamisme et vieillissement ", est une analyse théorique de type existentialiste et fait appel à la construction sociale du vieillissement. La deuxième partie, "À chacun d'inventer son vieillissement ", fait état de l'expérience de la retraite de l'auteure et de sa réflexion sur sa propre retraite. Dans l'ensemble la discussion est surtout réflexive, et c'est d'ailleurs la force et l'intérêt de ce livre.

La proposition initiale de l'auteure est de situer le vieillissement dans la dynamique du vivant. Le premier élément de réflexion est la prise de conscience de son vieillissement. La plupart des personnes ne se voient pas vieillir, car le discours sociétal identifie le vieillissement à la vieillesse. Un des écrans est la construction sociale du vieillissement. La société organise notre vie à travers ses institutions (famille, école, travail, etc.). L'âge chronologique sert donc de critère pour l'évaluation des événe- 